\title{
Modelo de Gestión por Competencias para optimizar el desempeño laboral de los colaboradores de la Municipalidad Provincial de Rioja, 2020
}

\author{
Jimmy Pacherrez Riva \\ rivapm@hotmail.com \\ Universidad Nacional de San Martín \\ ORCID:_0000-0002-8235-6371 \\ Gloria Marrufo Fernández \\ marrufofernandezg@gmail.com \\ Universidad César Vallejo \\ ORCID: 0000-0002-8784-1894
}

\section{RESUMEN}

El presente estudio tiene como objetivo proponer y validar un Modelo de gestión por competencias para optimizar el desempeño laboral de los colaboradores de la Municipalidad Provincial de Rioja, 2020. El estudio de investigación es de tipo aplicada de enfoque cualitativo, de diseño de investigación no experimental, descriptivo, propositivo. Como técnicas de recolección de datos se ha utilizado la encuesta y como instrumento cuestionarios por cada variable de estudio. Se concluyó que el desempeño laboral de los colaboradores de la Municipalidad Provincial de Rioja es deficiente, por lo que es importante diseñar e implementar un Modelo de Gestión por Competencias para desarrollar o fortalecer competencias cognitivas, habilidades y actitudinales optimizando su rendimiento, productividad y la administración de los recursos financieros y humanos.

Palabras clave: gestión; competencia; desempeño; optimizar. 


\title{
Competency Management Model to optimize the work performance of employees of the Provincial Municipality of Rioja, 2020.
}

\begin{abstract}
:
The objective of this study is to propose and validate a Competency Management Model to optimize the work performance of employees of the Provincial Municipality of Rioja, 2020. The research study is of an applied type of qualitative approach, of nonexperimental research design, descriptive, purposeful. The survey was used as data collection techniques and questionnaires for each study variable were used as an instrument. It was concluded that the work performance of the collaborators of the Provincial Municipality of Rioja is deficient, so it is important to design and implement a Competency Management Model to develop or strengthen cognitive skills, abilities and attitudinal optimizing their performance, productivity and administration of financial and human resources.
\end{abstract}

Keywords: Management; competition, performance, optimize.

Artículo recibido: 03 nov. 2020 Aceptado para publicación: 07 dic. 2020

Correspondencia rivapm@hotmail.com Conflictos de Interés: Ninguna que declarar 


\section{INTRODUCCIÓN}

En este mundo globalizado, las organizaciones están impulsando fuerzas laborales competitivas de habilidades que aplican en la identificación y satisfacción de los inconvenientes cotidianos que combaten en su costumbre laboral, por tal razón es que la rivalidad es con uno mismo, en busca de ser superiores, personal y profesionalmente; por esa razón a raíz de la globalización acarreó con su llegada renovadores enfoques para las instituciones públicas o privadas que tienen vínculo claro con la modificación en las organizaciones y en la creación del recurso humano por esto rastrean mayores y superiores expertos que les permita subir su competitividad y hacer mejor la eficacia. Un inconveniente mundial es la presencia de una alta tasa de desempleo laboral ya que, se vienen apareciendo inconvenientes para contemplar los cargos laborales gracias a la falta del perfil correspondiente para ocupar esas plazas laborales. Las instituciones públicas, fundamentalmente las municipalidades muestran más grande contrariedad para hallar a los expertos idóneos para los cargos disponibles. La carencia de capacidades en diferentes superficies de la plaza de trabajo es la pura realidad, es una circunstancia que va en aumento y que podría boicotear los mecanismos del desarrollo y las finanzas.

Es por ello que se realiza, la administración por competencias porque su evaluación facilita descubrir potenciales que hay adentro de las organizaciones o instituciones que desarrollan virtudes que están en competencia en el desarrollo insuperable de las ocupaciones en que se desenvuelven los ayudantes. La problemática que se presenta en la realidad social, está condicionada casualmente con los nuevos cambios mundiales, como manifestación de la mano del hombre y del mercado, se está viviendo la época de la sociedad del conocimiento, la nanotecnología facilita la relación momentánea y el ingreso a la información de forma ligera y en el mismo instante, descartando obstáculos y reduciendo trayectos entre individuos que radican en distintos sitios. En la actualidad la inclinación en las entidades públicas y privadas, es poseer un trabajador apreciado y operativo en su desarrollo laboral, con competencias propias del colaborador.

Los problemas en las municipalidades referidos al desempeño laboral son altos, puesto que muchos de los trabajadores están asumiendo cargos sin tener la experiencia ni el perfil profesional que se requiere para ejercer sus funciones, ocasionando que las relaciones interpersonales no sean las más optimas entre los colaboradores administrativos. Teniendo en cuenta los aspectos mencionados anteriormente y considerando que la 
satisfacción al usuario es un elemento fundamental en la evaluación de los servicios, por consiguiente, estos deben desarrollarse hacia niveles superiores, en donde el usuario se sienta totalmente complacido con el servicio brindado por los colaboradores de estas instituciones públicas.

En la Municipalidad provincial de Rioja, región de San Martín, se viene observando que, dentro de esa institución gubernamental, los funcionarios no están ejerciendo eficazmente sus habilidades laborales, porque hay una diferencia entre lo que saben y lo que hacen, frecuentemente, como resultado de sus actitudes, destrezas, acciones, práctica de valores, uso de capacidades nada aceptable ante la sociedad, además de una deficiencia de toma de elecciones y comunicación de información en el entorno laboral. Sumado a ello, no existe un apropiado proceso de selección y reclutamiento de personal; lo que origina que dichos profesionales se encuentren en un cargo en donde no son competentes; la causa viene a ser que no existe un programa de gestión por competencias que optimice la administración de los responsables que gerencian dicho municipio.

En consecuencia, se planteó el problema general ¿En qué medida un modelo de gestión por competencias optimizará el desempeño laboral de los colaboradores de la Municipalidad provincial de Rioja, 2020? En tal sentido se enunció los problemas específicos: ¿Cuál es el nivel de desempeño laboral de los colaboradores de la Municipalidad provincial de Rioja, 2020?, ¿Qué características tiene el desempeño laboral de los colaboradores?, ¿Cómo estará caracterizado la gestión por competencias de los funcionarios de la Municipalidad provincial de Rioja, 2020?„¿ Cuáles son las razones o nudos críticos que dificultan que los colaboradores tengan un buen desempeño laboral, ¿Cómo estará diseñado el modelo de gestión por competencias, ¿Cuánto es la valoración del modelo de gestión por competencias a través de juicio de expertos?.; en este sentido, esta investigación se justifica por conveniencia, porque con el modelo propuesto contribuirá a mejorar el desempeño laboral de los colaboradores de la municipalidad en estudio. La relevancia social, al mejorar las competencias laborales de los colaboradores, se mejorará la atención al usuario generando satisfacción por el servicio recibido. Con respecto a las implicancias prácticas, se busca contribuir aportando una serie de recomendaciones sobre la gestión por competencias que puedan servir para desarrollar efectividad organizacional, tomando en cuenta factores e indicadores en el proceso, tales como la comunicación interna, la planificación, entre otros que ayuden a mejorar el 
desarrollo organizacional. Se busca que esta investigación aporte transcendentalmente a la reducción de costos, de cuellos de botella que generan pérdidas de dinero y tiempo. En cuanto al valor teórico, se contribuirá con información científica sobre el desempeño laboral y se diseñará un modelo de gestión por competencias, las que serán validados por juicio de expertos, el mismo que podrá implementarse en otras instituciones ediles. En cuanto a la utilidad metodológica, aportará con la estandarización del instrumento sobre el desempeño laboral.

Como objetivo general se enuncia de la siguiente manera: Proponer un modelo de gestión por competencias para optimizar el desempeño laboral de los colaboradores de la Municipalidad provincial de Rioja, 2020. Asimismo, se planteó los objetivos específicos: (i) Identificar el nivel del desempeño laboral de los colaboradores de la Municipalidad provincial de Rioja, 2020. (ii) Caracterizar el desempeño laboral de los colaboradores de la Municipalidad provincial de Rioja, 2020. iii) Caracterizar la gestión por competencias de los funcionarios de la Municipalidad provincial de Rioja, 2020, (iv) Diseñar el modelo de gestión por competencias. (v) Conocer la valoración del modelo de gestión por competencias a través de juicio de expertos. Finalmente, se formuló la hipótesis de investigación: si diseñamos un modelo de gestión por competencias, entonces se podrá optimizar el desempeño laboral de los colaboradores de la Municipalidad provincial de Rioja, 2020.

Los trabajos previos relacionados a la variable de estudio encontrados a nivel internacional como de, Hernández, V. (2018), Modelo de administración de talento humano por competencias de la ONG para la discapacidad fundación cuesta Holguín. (Artículo científico). Pontificia Universidad Católica de Ambato, Ecuador. Tipo de estudio no experimental, diseño descriptivo-propositivo, muestra 23 personas, técnica encuesta, instrumento cuestionario, llegó a la conclusión, el modelo de administración de talento humano apoyado en un sistema de competencias e indicadores para entidades sin ánimo de lucrar que atienden a personas con discapacidad diseñado, se enfoca en el avance de las competencias organizacionales y de puestos claves, base para la incorporación de los subsistemas de selección, formación-desarrollo, compensación y evaluación del desarrollo. De igual modo, ayuda a alinear los objetivos organizacionales, dando permiso un rastreo y control de los indicadores propuestos, lo que contribuirá a 
hacer mejor la calidad de la atención que se ofrece a la gente con discapacidad, y a la más grande inserción socio-laboral de las mismas en las compañías privadas y públicas.

Por su parte, Grijalva, M. Castro, J. y Guamán, M. (2019), Desempeño laboral como vector ocupacional. (Artículo científico). Universidad Técnica de Ambato, Ecuador. Estudio cuantitativo, no experimental, muestra 53 empleados, técnica la encuesta, instrumento cuestionario. Concluyó, la actitud, personalidad, percepción y aprendizaje están asociadas con el desempeño laboral. La calidad de trabajo, influye en el nivel de desempeño de los trabajadores y desarrolla la predisposición para cumplir sus tareas, mientras que el trabajo en equipo es un factor significativo para el desarrollo de habilidades e innovación. Sin embargo, en la empresa el individualismo, afecta el clima organizacional y la satisfacción laboral donde está implicada la remuneración.

En cambio, Reinoso, M. y Pérez, J. C. (2019), Cultura organizacional y desempeño laboral en los colaboradores de las PYMES. (Artículo científico). Universidad Autónoma de Chapingo. México. Tipo de estudio correlacional, diseño no experimental, muestra 35 personas, técnica encuesta, instrumento cuestionario, llegaron a la conclusión, que, la actitud para ofrecer un producto y un servicio de calidad requiere que los trabajadores brinden una buena atención al cliente, que exista la predisposición para realizar sus actividades con eficiencia cumpliendo asertivamente con los procedimientos para el empleado y la Administración. Desde el punto de vista competitivo los trabajadores de la empresa pueden adaptarse a los cambios con total normalidad. Puesto que la innovación permite dotarse de recursos técnicos mejorar y modernizar la presentación del producto, infraestructura y el servicio.

Para, Correa, St. (2017), Incidencia de la Gestión por competencias en el crecimiento empresarial. (Artículo científico). Universidad Técnica de Machala, Ecuador. Diseño de investigación descriptivo, muestra 28 profesionales, técnica encuesta, instrumento cuestionario, concluyó, la aplicación de la administración por rivalidad en las compañías exportadoras, tuvo un efecto positivo en el talento humano, ya que se ha verificado una optimización en sus habilidades y capacidades, por lo tanto, mayor crecimiento en sus ventas y mejor rentabilidad.

Asimismo, Santacruz, L. (2019), Análisis de modelos de gestión por competencias como fomento a la competitividad en organizaciones del sector textil. (Artículo científico). Universidad CES, Medellín, Colombia. Tipo de investigación básica, diseño descriptivo, 
muestra 120 trabajadores, técnica encuesta, instrumento cuestionario, concluyó, que, los modelos de gestión por competencias hacen una incorporación y articulación entre los procesos de selección, evaluación del desarrollo y capacitación; con el objetivo de que las organizaciones logren administrar, potencializar y desarrollar acertadamente su talento humano, debido que son tomadas como "una creación desde una conjunción de elementos (conocimiento, entender llevar a cabo, características o aptitudes), y elementos del ámbito (relaciones, documentos, aclaraciones y otros) que son movilizados para conseguir un buen desarrollo.

Por su parte, Oliva, Y. (2019), Gestión por competencias. Elementos que limitan su aplicación. (Artículo científico). Universidad del Oriente, Venezuela. Tipo de estudio no experimental, diseño descriptivo, muestra 23 gerentes de empresas afines, técnica entrevista, instrumento cuestionario, concluyó; la aplicación de las diferentes herramientas permitió realizar un diagnóstico estratégico de COMETAL UEB Santiago y definición de elementos esenciales; se demostró que algunas de las debilidades definidas en la UEB, constituyen los elementos que limitan la gestión por competencias en dicha organización.

Con respecto a los trabajos previos a nivel nacional, se encontró a Díaz, J. (2018), Influencia de las competencias gerenciales y la gestión por resultados en la imagen institucional. (Artículo científico). Universidad César Vallejo, Lima, Perú. Investigación no experimental, diseño transversal, la muestra fue de 218 de una población 500, técnica encuesta, instrumento cuestionario, concluyó, que, las competencias gerenciales y la administración por resultados influyen de forma positiva en el accionar y la personalidad corporativa; puesto que la efectividad predictiva de la posibilidad de ocurrencia de las categorías de la variable ligado es efectiva. Es decir, en la empresa hay un liderazgo por parte del gerente, esto se evidencia en el cumplimiento de metas y en el incremento de la productividad.

Asimismo, Nuñez, N. (2017), Perfil por competencias gerenciales en directivos de instituciones educativas. (Artículo científico). Universidad Católica Santo Toribio de Mogrovejo, Chiclayo Perú. Estudio no experimental, diseño descriptivo, muestra fue de 340 de una población de 71132 docentes de tres UGEL, técnica encuesta, instrumento cuestionario, concluyó, que, algunas invariantes en los desempeños requeridos en el perfil de los directivos de las instituciones educativas requieren tener un óptimo manejo de 
dirección, que se requiere para el emprendimiento de procesos de administración escolar académicos y administrativos; falta un liderazgo, con idea, imaginación, perspectiva de futuro y rígidos valores en el desarrollo personal y profesional; la administración del cambio, no tiene ocupaciones y proyectos innovadores para lograr las misiones institucionales con planeación, objetivos estratégicos establecidos, compromiso y abordando diferentes contextos en el marco del mejoramiento continuo de la institución educativa.

Al respecto, Carhuachín, F. (2018), Administración por competencias para el desarrollo laboral del talento humano en la Municipalidad provincial de Otuzco - La Independencia, 2018. (Artículo científico). Universidad Señor de Sipán. Pimentel, Perú. Tipo de investigación básica-descriptivo, diseño no experimental, población y muestra 21 servidores públicos, técnica observación, instrumento cuestionario. Concluyó, que, no se está aplicando una administración por competencias, por lo cual se sabe que un modelo de administración por competencias optimización relevantemente el desarrollo laboral del talento humano. El desarrollo laboral del talento humano, no es evaluado en forma diaria y se hace primordial la utilización de un modelo de administración por competencias incidiendo en el correcto perfil profesional y el fortalecimiento de sus competencias laborales para hacer mejor el desarrollo laboral del talento humano en la municipalidad.

Mandamiento, A. (2019), Administración por competencias y desarrollo laboral del personal de la Municipalidad provincial de Candarave. (Artículo científico). Universidad Privada de Tacna, Perú. Tipo de investigación básico, diseño correlacional, población y muestra 42 colaboradores, técnica encuesta, instrumento cuestionario, concluyó, con un nivel de confianza del 95\% que existe relación directa y positiva, entre la selección de personal y la gestión por competencias del personal. Asimismo, la fuerza de la relación es de 0.79 , indicando una fuerte relación.

Con respecto a los trabajos previos a nivel de la región y/o local; solo se encontró tesis como de, Lozano, G. y Mendoza, M. (2018), Relación de la administración por competencias con el desarrollo laboral de los ayudantes en una municipalidad de la zona San Martín - Perú. (Tesis de posgrado). Universidad Peruana la Unión. Tarapoto, Perú. El estudio fue básico, diseño correlacional, la población y muestra 50 colaboradores, técnica encuesta, instrumento cuestionario, concluyó; que, existe relación importante 
entre las cambiantes de estudio, o sea, a mejor avance de administración por competencias, mejor va a ser el nivel de desarrollo laboral, el nivel de efectividad, eficacia, la eficacia y el nivel de relaciones entre personas entre los ayudantes de la institución.

Finalmente, Bocanegra, J. (2018), Competencias expertos requeridas por las compañías en Tarapoto. (Artículo científico). Universidad Peruana Unión, Tarapoto, Perú. Diseño de estudio descriptivo, muestra 108 de una población de 180 empresas, técnica la encuesta, instrumento cuestionario, concluyó, que, en las empresas en Tarapoto las competencias que requieren en los trabajadores son la empatía, naturalidad y liderazgo; seguido de los valores, que es la competencia más requerida; en consecuencia, un trabajador debe poseer actitudes que inspiren confianza que practiquen un criterio ético en el desarrollo de sus actividades. En cuanto a las técnicas de reclutamiento, no existe; ya que, en la mayoría de las instituciones públicas y privadas el reclutamiento de personal que más se utiliza, es la presentación de personas por recomendación para ocupar cargos de confianza y otros puestos.

Las teorías relacionadas al desempeño laboral, es definido por Mondy (2010) a todos los procesos de la organización que determinan el nivel de calidad con el que los empleados y los equipos ejercen sus funcionalidades. (p. 25); por su parte, Palaci (2005) conceptualizó, "El desarrollo laboral es el valor que se estima dar a la organización de los distintos episodios conductuales que un sujeto transporta a cabo en un lapso de tiempo" (p.155). Además, Chiavenato (2013) complementó: "El desarrollo laboral es el esfuerzo que pone cada sujeto en funcionalidad del valor de las recompensas ofrecidas; comandado por las habilidades y capacidades del sujeto y por las percepciones que él tiene del papel que está realizando (pp.260-261).

En cambio, Matos (2013) conceptualizó el desempeño laboral como "la eficiencia que demuestra el colaborador en el trabajo; cuyo resultado será medido a través de un control y evaluación pertinente. Por su parte, Quintero (2012) detalló que, el desarrollo de los trabajadores debe estar en funcionalidad al cumplimiento de las reglas y normas de la compañía, donde se tendría que continuar los métodos establecidos para hacer las ocupaciones. Es donde el trabajador exhibe sus capacidades y conocimiento para el avance eficaz de los trabajos en la compañía (p. 121). 
Entre los factores que influyen en el desempeño laboral, para, Pons (2012) afirmó que la razón es un aspecto sustancial que influye en el desarrollo laboral así sea de parte de la compañía, por parte del trabajador y la económica. Estos componentes son: adecuación / ámbito de trabajo: Es muy sustancial sentirse práctico en el sitio de trabajo dado que esto nos ofrece superiores opciones de llevar a cabo nuestro trabajo de manera correcta. Lugar de objetivos: El lugar de objetivos es una aceptable técnica para motivar a los empleados. Reconocimiento del trabajo: El reconocimiento del trabajo efectuado se encuentra dentro de las técnicas más destacables. La participación del empleado: Participa en el control y idealización de sus tareas sintiéndose con más seguridad y formando parte de la compañía. La formación y avance profesional: Se sienten motivados por su desarrollo personal y profesional, de forma que beneficiar su formación (pp. 122- 123).

En cuanto, a los procedimientos de evaluación basados en el desarrollo laboral, según, Sales (2002) son: Autoevaluaciones: Puede constituir una técnica de gran ayuda, cuando el propósito es alentar el avance individual. Gestión por objetivos: El supervisor como el empleado establecen en conjunto los objetivos de desarrollo deseables. Evaluaciones psicológicas: Los psicólogos emplean las evaluaciones, en funcionalidad a la evaluación del potencial del sujeto y no su desarrollo previo. Procedimientos de los centros de evaluación: Forma estandarizada para considerar a de los empleados, argumentándose en tipos múltiples de evaluación y múltiples evaluadores.

Según, Werter (2012) explica que el desempeño laboral a menudo necesita ser evaluado con base en el desempeño de cada trabajador, de esta manera se alcanzarían los objetivos de la organización (p. 33). Por otro lado, Ramírez (2016) explicó que la evaluación del desempeño del personal debe llevarse a cabo con la implementación de diferentes estrategias, de esta forma se obtendrían resultados mejores y más precisos con los que se puedan establecer medidas correctivas que puedan generar mayor valor para las actividades organizacionales. Dicho desempeño debe estar en función del cumplimiento de la misión de la empresa y el logro de la visión.

Por su parte, Dessler (2001) identificó algunos de los factores que se tienen en cuenta para la evaluación de los trabajadores, en este caso es a través del monitoreo, también existe la evaluación de cada uno de los trabajadores, así como la autoestima que es crucial para un buen desempeño. De esta manera, habría información, como resultado de la 
evaluación de la persona, que permita corregir las medidas para las deficiencias identificadas.

Las técnicas de evaluación del desempeño, de acuerdo con Urquijo y Bonilla (2008), pueden proporcionar varias técnicas para evaluar el desempeño de los trabajadores como una jerarquía de trabajadores en una lista, que se basaría en un mejor desempeño y un desempeño más bajo. Otra técnica podría ser comparar actividades con sus colegas en la misma área, de esta manera sabría quién tiene mejores resultados. También hay una escala de evaluación, que consiste en una evaluación mediante la calificación de diferentes escalas, esto puede ser realizado por los gerentes inmediatos de cada área de la empresa. Del mismo modo, se puede utilizar una lista de verificación de actividad, de esta manera se puede evaluar el desempeño de un empleado (p. 32).

Por su parte, Chiavenato (2013) Explicó que los beneficios de la evaluación del desempeño se otorgan al individuo, el gerente, la organización y la comunidad. Estos beneficios para el gerente serían al evaluar el comportamiento de cada trabajador en la empresa, de esta manera él tendría información actualizada sobre sus empleados para poder establecer estrategias de solución para todos los problemas que surgen en las áreas de trabajo, de modo que pueda transmitir la importancia del trabajador a evaluar al personal para mejorar su desempeño. Los beneficios para los subordinados serían que conocerían las reglas de trabajo y lo que esperan de ellos, también sabrán cuál es su índice de desempeño y las medidas que los gerentes tomarían para mejorar su desempeño en la empresa. En los beneficios para la organización, los resultados que se alcanzarían se basan en el desempeño de los trabajadores $\mathrm{y}$, por lo tanto, cumplen con los objetivos establecidos para el desarrollo de la organización (p. 51).

Mientras que, Muñoz (2000) define la satisfacción laboral como un sentimiento de placer o positivo que un sujeto experimenta al realizar un trabajo que le interesa, en un entorno que le permite sentirse cómodo, en el marco de una empresa u organización que es atractiva y para aquellos que reciben una serie de psicópatas. -compensación socioeconómica de acuerdo con sus expectativas. Del mismo modo, define la insatisfacción laboral como un sentimiento de insatisfacción o negativo que experimenta un sujeto porque está haciendo un trabajo que no le interesa, en un entorno en el que no está satisfecho dentro de una empresa u organización que no es atractiva para él y por el 
cual recibe una serie de compensaciones psico-socioeconómicas que no cumplen con sus expectativas (p. 45).

En cambio, para, Loitegui (1990), los conceptos de actitudes, satisfacción y moral del trabajo son muy similares y están altamente interrelacionados, pero de ninguna manera pueden considerarse idénticos. La actitud puede contribuir a la satisfacción, que se compone de un conjunto de ellos y, a su vez, la satisfacción influye en la moral. Este autor propone las siguientes definiciones: (i) Actitud en el trabajo: es la forma en que el trabajador se siente en relación con su trabajo y su disposición a reaccionar de manera especial a los factores relacionados con ese trabajo. (ii) Satisfacción laboral: es el resultado de las diversas actitudes que tiene el trabajador hacia su trabajo, los factores relacionados con él y la vida en general. (iii) Moralidad en el trabajo: desde el punto de vista del trabajador, consiste en tener un sentimiento de ser aceptado y pertenecer al grupo cuando se une a los grupos. (p. 121).

De manera similar, Palma (2001) presentó los siguientes factores que favorecen la satisfacción laboral de los empleados: (a) condiciones físicas, (b) compensación, (c) política administrativa, (d) relaciones sociales, (e) desarrollo personal, (f) el desempeño de las tareas. y (g) la relación con la autoridad. Peiró (1996) descubrió que, en la actualidad, se presta más atención a la calidad de la vida laboral en contraste con años anteriores donde solo se buscaba la calidad en el desempeño. (pp. 77-81).

Dimensiones del desempeño laboral, para, Da Silva, (2008), tiene tres: (1) Dimensión eficiencia, que significa "trabajar para que los recursos se utilicen de manera más adecuada" (p. 32). De manera similar, Mokate (2002) definió la eficiencia como "la capacidad de seleccionar y usar los materiales más eficientes y menos desechables para realizar una tarea o un propósito". (pág. 59). En otras palabras, la eficiencia es la relación entre el trabajo realizado, el tiempo invertido, la inversión realizada para hacer algo y el resultado logrado. (2) Dimensión eficacia, es el nivel de alcanzar metas y objetivos. La efectividad se refiere a nuestra capacidad de lograr lo que pretendíamos hacer. Este significado es reforzado por Mokate (2002), quien indicó que la eficiencia es el cumplimiento de las metas establecidas durante el tiempo planificado y con la calidad esperada (p. 23). Lo que significa ser efectivo es trabajar para lograr los objetivos de la organización de acuerdo con el protocolo de calidad de acuerdo con los plazos establecidos, ya sea a corto, mediano o largo plazo. 
En cuanto a la dimensión (3) Relación interpersonal, según Bisquerra (2003), "es una interacción mutua entre dos o más personas". Estas son las relaciones sociales como tales gobernadas por las leyes e instituciones de interacción social (p. 23); Según la opinión de Fernández (2003: 25), "trabajar en un ambiente de trabajo óptimo también es extremadamente importante para los empleados, ya que un ambiente saludable afecta directamente su desempeño y bienestar emocional". (p. 25), por lo tanto, las relaciones interpersonales son un factor muy importante en ciertas áreas de trabajo donde es necesario el trabajo en equipo o el desarrollo de la cartera de clientes. Esta es la razón por la cual las personas con las que es fácil relacionarse y pasar tiempo tienden a tener mejores opiniones para ciertos puestos que aquellas que prefieren trabajar individualmente.

Las teorías relacionadas a la gestión por competencias, para Manríquez (2012) Las competencias son esenciales para que los seres humanos funcionen eficientemente en su vida diaria y, a su vez, pueden ser competitivas en un entorno en constante cambio y evolución. En comparación con Pérez (2012), afirma que la única forma para que las personas logren un desarrollo adecuado es saber qué habilidades tienen y con qué actitudes realizan sus tareas diarias (p. 16).

"Las competencias son consecuencia de una integración de actitud coherente (visión, misión, filosofía); habilidades (rutinas, políticas, aprendizaje); habilidades (liderazgo, habilidades, experiencias); permitiéndoles interactuar internamente y de manera extremadamente efectiva, favoreciendo su personalización y facilitando el desempeño de habilidades técnicas y personales" (González, 2000, p.42).

Massó y Lozares (2012) muestra que el conocimiento, la creación de conocimiento, el conocimiento son parte de una mezcla de conocimiento, habilidades y experiencias que son perceptibles en el desarrollo de las personas de acuerdo con el entorno en el que se encuentran y, por lo tanto, en un contorno específico para la competencia está especialmente demostrado más que en la aplicación de los mismos porque depende mucho de dónde, cómo y con qué herramientas se realiza (p. 211).

En relación con Manríquez (2012), demuestra cuatro fases del proceso de determinación de que normalmente se desarrolla una competencia. Los primeros procesos, basados en una competencia, muestran una interacción clara con los procesos cognitivos y posteriores con el comportamiento. En relación con lo que se ha enfatizado en el pasado, las compañías necesitan promover la gestión del conocimiento y aplicar un modelo de 
fuerza laboral, donde la mayoría de sus miembros conocerán a fondo su perfil de puesto y se adaptarán a las actividades en el trabajo en el que se desempeña, el propósito es adaptar un perfil basado en habilidades de acuerdo con lo que la organización requiere para lograr sus objetivos (Sandoval y Pernalete, 2014, p. 55).

La gestión basada en competencias se define como una herramienta para el talento humano, con una propuesta específica basada en los resultados de los empleados en un proceso de gestión dado y, a su vez, alineada sinérgicamente con los resultados de la empresa. Todo esto se enmarca en instrumentos para evaluar e identificar competencias (Guerrero, Valverde Aparicio y Tatiana, 2013, p. 81).

Por lo tanto, Chávez (2012) asegura que la importancia de la gestión de la competencia se basa en criterios que conducen a la gestión adecuada de las personas, en función de las habilidades que contribuyen al logro de los objetivos de la organización. (p. 33), por otro lado, para García, García, Pérez, González y Martínez (2014), la gestión de competencias en el campo organizacional, enfatiza el compromiso de cada uno de sus miembros, quienes proponen un sistema eficiente en la dirección de capacidad innovadora y creativa bajo la estructura de trabajo social y empresarial en la que opera, siempre y cuando se base en las habilidades del equipo (p. 18). En resumen, su aplicación en diferentes organizaciones ha ganado un valor significativo en términos de requisitos del mercado, tratando de desarrollar e implementar un equipo adecuado a las necesidades, de modo que la selección de talentos humanos se convierta en una tarea ardua, con respecto a las habilidades y el conocimiento logrando así una ubicación correcta en sus respectivas áreas para cada miembro de la organización (Chávez, 2012, p. 35)

Para, García, Reyes, y Carballo (2009), la implementación de un modelo de gestión de competencias implica, entre otras cosas, un cambio cultural en la forma en que las organizaciones evalúan el conocimiento (capturarlo, seleccionarlo, organizarlo, distinguirlo y presentarlo) y priorizar el aprendizaje de su propia experiencia. y con un enfoque en la obtención, recopilación y uso del discernimiento para resolver dificultades y desarrollar la inteligencia y adaptabilidad de la empresa. Por otro lado, para Tejada y Navío (2005), la aplicación de un modelo de gestión de la competencia en las empresas ofrece una serie de ventajas, entre las que destacan las siguientes: destacar el talento humano como la clave para el desarrollo y el tiempo de la organización vida de la empresa La misma. Pase de roles y puestos bien definidos a gestión integrada de procesos y trabajo 
en equipo. Colabore bajo el modelo de competencia para que sus empleados se adapten rápidamente a los cambios permanentes que ocurren en el mercado competitivo. Promueva una mentalidad de aprendizaje constante. Proporcione a los miembros de la organización nuevos conocimientos que puedan aplicarse como recompensa por su dedicación y esfuerzo.

¿La gestión adecuada de las habilidades promoverá el crecimiento empresarial? Según Dorta y Verona (2006), el crecimiento depende de varios factores que pueden ser internos, como la edad y el tamaño, la motivación, la estructura de propiedad y la gestión del conocimiento, y entre los factores externos puede estar el entorno superior o macro. Sin embargo, Rojo y González (2007) confirman que las necesidades que surgen en las empresas con respecto a la supervivencia del mercado se mencionan en el factor de habilidad que siempre ha tratado de mantener y reconocer. Basa su apoyo para tal situación en el crecimiento interno y externo de la compañía. Por lo tanto, la gestión de la competencia se postula como una herramienta fundamental para que las empresas crezcan y se adapten a los cambios que se producen en el entorno, a través de la gestión adecuada del talento humano (García, Reyes y Carballo, 2009).

Entre sus dimensiones, tenemos tres: (1) Dimensión selección del personal, se define como elegir al individuo adecuado para el puesto correcto o, en un sentido más amplio, elegir entre los candidatos reclutados para el puesto más adecuado, para ocupar los puestos existentes en la empresa, tratando de mantener o aumentar la eficiencia y el desempeño del personal. El criterio de selección se basa en los datos y la información mantenida en relación con el puesto que se proporcionará. (Werther y Davis, 2000).

(2) La dimensión de capacitación y desarrollo, es el conjunto de acciones de preparación continua y planificada, concebida como una inversión desarrollada por organizaciones que tienen como objetivo mejorar las habilidades y calificaciones de los trabajadores, cumplir las funciones del puesto con calidad, cumplir con la calidad. Las funciones del puesto, garantizan su desempeño exitoso y alcanzan los máximos resultados de producción o servicio (Bailón Olea, 2014).

(3) La carrera profesional, está vinculada al desarrollo de las personas dentro de una organización, varios factores (nuevas tecnologías, flexibilidad en el empleo, cambios en los estilos de producción) llevan a las personas a tener que adaptarse constantemente a 
nuevas situaciones relacionadas con las áreas ocupacionales y laborales (desempleo, mantenerse al día con nuevos desarrollos, etc.). (Champagnet, 2001).

Méndez (2000) y Pontifes, (2003) fueron los pioneros en usar el concepto de evaluación del desarrollo a comienzo del siglo XIX en Escocia. Asignaron un supervisor a cada trabajador para hacer notas de su desarrollo y conceder colores en concordancia al nivel de desempeño que reportaban en consideración con el puesto ocupado. Además, usaron procedimientos estadísticos para su consideración.

Maristany (2015) la evaluación del desempeño nació a inicios del siglo XX en USA y se aplicó por primera oportunidad a los comerciantes contratados por el gobierno. De la misma manera fue aplicado al batallón de ese país en 1916. Este mismo creador acotó que en 1923 se estableció en el Acta de Categorización, la manera en que cómo debía hacerse la evaluación en todo el gobierno de ese país. Con el paso de los años la evaluación del desarrollo se convirtió en el procedimiento de acompañamiento para los empleados que no alcanzan los estándares de cada compañía y para medir el buen desempeño en cada puesto del sector productiva, así como es servible para considerar la consideración del sistema de capacitación que usa la organización, no olvidando que los trabajadores necesitan una realimentación desde los resultados que consiguen dado que al comprender su nivel de eficacia tienen la posibilidad de sentirse seguros y tomados presente por la compañía a la que sirven (Méndez, 2000).

\section{ESTRATEGIAS METODOLÓGICAS O MATERIALES Y MÉTODOS.}

Esta investigación fue de tipo aplicada, porque se basó fundamentalmente a recomendar estrategias de gestión por competencias para mejorar el desempeño laboral de los colaboradores de la Municipalidad Provincial de Rioja. (Concytec, 2018)

La población y muestra estuvo conformada por 39 colaboradores nombrados y 9 directivos y/o jefes de área de la Municipalidad provincial de Rioja.

Como técnica se utilizó la encuesta y como instrumento los cuestionarios sobre aspectos del desempeño laboral en las dimensiones de eficacia, eficiencia y relaciones interpersonales. En cuanto a la gestión por competencias se tomó en cuenta las siguientes dimensiones: Selección de personal, capacitación y desarrollo y carrera profesional.

\section{RESULTADOS Y DISCUSIÓN}

\section{RESULTADOS}

El resultado arrojado de la investigación se obtuvo por la aplicación del cuestionario a los trabajadores nombrados y directivos y/o jefes de área de la municipalidad, y al mismo 
tiempo, se realizó el análisis e interpretación de la información obtenida. Se mantuvo de manera cerca los objetivos planteados.

\section{Tabla 1}

\section{Nivel del desempeño laboral de los colaboradores}

\begin{tabular}{|c|c|c|c|c|c|c|}
\hline \multirow{2}{*}{ Nivel } & \multirow{2}{*}{\multicolumn{2}{|c|}{ Escala }} & \multicolumn{2}{|c|}{ Funcionario } & \multicolumn{2}{|c|}{ Colaborador } \\
\hline & & & fi & $\%$ & fi & $\%$ \\
\hline Bajo & 15 & 34 & 7 & $77.8 \%$ & 26 & $66.7 \%$ \\
\hline Medio & 35 & 54 & 2 & $22.2 \%$ & 13 & $33.3 \%$ \\
\hline Alto & 55 & 75 & 0 & $0.0 \%$ & 0 & $0.0 \%$ \\
\hline Total & & & 9 & $100.0 \%$ & 39 & $100.0 \%$ \\
\hline
\end{tabular}

Fuente: Cuestionario aplicado a funcionarios y colaboradores de la municipalidad provincial Rioja.

\section{Interpretación:}

En la tabla 1, se observa que los funcionarios perciben que el desempeño laboral de los colaboradores es "Bajo" en un 77.8\% (7), seguido de "medio" en un 22.2\% (2).

Asimismo, desde la percepción de los colaboradores ubican en su mayoría que el nivel de desempeño tiene un nivel "bajo" en un 66,7\% (26) y un nivel "medio" en 33,3\% (13).

\section{Tabla 2}

Desempeño laboral de los colaboradores desde la percepción del funcionario

\begin{tabular}{|c|c|c|c|c|c|c|c|}
\hline \multirow{2}{*}{ Indicadores } & & \multicolumn{2}{|c|}{ TED /ED } & \multicolumn{2}{|l|}{$\mathbf{I}$} & \multicolumn{2}{|c|}{ DA / TDA } \\
\hline & & fi & $\%$ & fi & $\%$ & fi & $\%$ \\
\hline \multirow{6}{*}{ Eficacia } & $\begin{array}{l}\text { Cumplen con los objetivos planificados } \\
\text { en el POI. }\end{array}$ & 9 & $100.0 \%$ & 0 & $0.0 \%$ & 0 & $0.0 \%$ \\
\hline & $\begin{array}{l}\text { Cuentan con recursos necesarios para } \\
\text { cumplir con los objetivos planificados } \\
\text { en el POI. }\end{array}$ & 9 & $100.0 \%$ & 0 & $0.0 \%$ & 0 & $0.0 \%$ \\
\hline & $\begin{array}{l}\text { Se socializa al inicio del año los } \\
\text { objetivos planificados en el POI. }\end{array}$ & 1 & $11.1 \%$ & 1 & $11.1 \%$ & 7 & $77.8 \%$ \\
\hline & $\begin{array}{l}\text { Se ha socializado el MOF, ROF, RIT, } \\
\text { MAPRO u otros. }\end{array}$ & 9 & $100.0 \%$ & 0 & $0.0 \%$ & 0 & $0.0 \%$ \\
\hline & Cumplen con las funciones establecidas. & 5 & $55.6 \%$ & 0 & $0.0 \%$ & 4 & $44.4 \%$ \\
\hline & $\begin{array}{l}\text { Considera que se ha incrementado la } \\
\text { productividad de los colaboradores en } \\
\text { este año. } \\
\text { Existen incentivos para motivar al } \\
\text { colaborador. }\end{array}$ & 8 & $100.0 \%$ & 1 & $11.1 \%$ & 0 & $0.0 \%$ \\
\hline
\end{tabular}




\begin{tabular}{|c|c|c|c|c|c|c|c|}
\hline & $\begin{array}{l}\text { Cumplen sus metas en función al puesto } \\
\text { y al tiempo establecido. }\end{array}$ & 7 & $77.8 \%$ & 0 & $0.0 \%$ & 2 & $22.2 \%$ \\
\hline & $\begin{array}{l}\text { Se asigna en el tiempo establecido los } \\
\text { recursos para el cumplimiento de las } \\
\text { actividades del POI. }\end{array}$ & 5 & $55.6 \%$ & 0 & $0.0 \%$ & 4 & $44.4 \%$ \\
\hline \multirow[t]{4}{*}{ Eficiencia } & $\begin{array}{l}\text { Se racionaliza los recursos solicitados de } \\
\text { cada área según su POI. }\end{array}$ & 8 & $88.9 \%$ & 0 & $0.0 \%$ & 1 & $11.1 \%$ \\
\hline & $\begin{array}{l}\text { Cumplen con los plazos establecidos en } \\
\text { el TUPA. }\end{array}$ & 9 & $100.0 \%$ & 0 & $0.0 \%$ & 0 & $0.0 \%$ \\
\hline & $\begin{array}{l}\text { Se encuentran comprometidos en las } \\
\text { actividades que realizan. }\end{array}$ & 9 & $100.0 \%$ & 0 & $0.0 \%$ & 0 & $0.0 \%$ \\
\hline & $\begin{array}{l}\text { Observa que el ambiente de trabajo es } \\
\text { agradable para los colaboradores. }\end{array}$ & 6 & $66.7 \%$ & 0 & $0.0 \%$ & 3 & $33.3 \%$ \\
\hline \multirow[t]{2}{*}{$\begin{array}{l}\text { Relación } \\
\text { interpersonal }\end{array}$} & $\begin{array}{l}\text { Existe una adecuada comunicación de } \\
\text { los colaboradores. }\end{array}$ & 0 & $0.0 \%$ & 0 & $0.0 \%$ & 9 & $100.0 \%$ \\
\hline & $\begin{array}{l}\text { La comunicación que se realiza entre } \\
\text { colaboradores contribuye a mejorar las } \\
\text { relaciones interpersonales. }\end{array}$ & 5 & $55.6 \%$ & 2 & $22.2 \%$ & 2 & $22.2 \%$ \\
\hline
\end{tabular}

Fuente: Cuestionario aplicado a funcionarios de la municipalidad provincial Rioja.

\section{Interpretación:}

En la tabla 2 se caracteriza el desempeño laboral de los colaboradores desde la percepción del funcionario, evidenciándose que, en la dimensión eficacia, el 100\% (9) están "Totalmente en desacuerdo" o "desacuerdo", que los colaboradores cumplan con los objetivos planificados en el POI, que cuenten con recursos necesarios para cumplir con estos objetivos, que se haya socializado al inicio del año el MOF, ROF, RIT, MAPRO u otros y que existan incentivos para motivar al colaborador, respectivamente. Asimismo, el 88,9\% (8) de los funcionarios considera que no se ha incrementado la productividad de los colaboradores en este año, ni cumplen con las funciones establecidas en un 55,6\% (5). En la dimensión eficiencia, los funcionarios indican estar "totalmente en desacuerdo" o "desacuerdo" en un 100\% (9) que los colaboradores cumplen con los plazos establecidos en el TUPA, que se encuentran comprometidos en las actividades que realizan. Por otro lado, el $88.9 \%$ (8) observa que no se racionaliza los recursos solicitados de cada área según su POI, que no se cumplen sus metas en función al puesto y al tiempo establecido $77.8 \%$ (7) y que no se asigna en el tiempo establecido los recursos para el cumplimiento de las actividades del POI, 55.6\% (5) respectivamente.

En la dimensión interpersonal, los funcionarios están "totalmente en desacuerdo" o "desacuerdo" en un $66.7 \%$ (6), que el ambiente de trabajo es agradable para los 
colaboradores y que la comunicación que se realiza entre ellos contribuye a mejorar las relaciones interpersonales en un 55.6\% (5).

\section{Tabla 3}

Desempeño laboral de los colaboradores desde la percepción del colaborador

\begin{tabular}{|c|c|c|c|c|c|c|c|}
\hline \multirow{2}{*}{ Indicadores } & & \multicolumn{2}{|c|}{ TED /ED } & \multicolumn{2}{|l|}{ I } & \multicolumn{2}{|c|}{ DA / TDA } \\
\hline & & fi & $\%$ & fi & $\%$ & fi & $\%$ \\
\hline \multirow{7}{*}{ Eficacia } & $\begin{array}{l}\text { Cumple con los objetivos planificados } \\
\text { en el POI. }\end{array}$ & 29 & $74.4 \%$ & 5 & $12.8 \%$ & 5 & $12.8 \%$ \\
\hline & $\begin{array}{l}\text { Cuenta con los recursos necesarios para } \\
\text { cumplir con los objetivos planificados } \\
\text { en el POI. }\end{array}$ & 27 & $69.2 \%$ & 4 & $10.3 \%$ & 8 & $20.5 \%$ \\
\hline & $\begin{array}{l}\text { Le socializaron al inicio del año los } \\
\text { objetivos planificados en el POI. }\end{array}$ & 21 & $53.8 \%$ & 7 & $17.9 \%$ & 11 & $28.2 \%$ \\
\hline & $\begin{array}{l}\text { Le socializaron el MOF, ROF, RIT, } \\
\text { MAPRO u otros. }\end{array}$ & 33 & $84.6 \%$ & 7 & $17.9 \%$ & 3 & $7.7 \%$ \\
\hline & $\begin{array}{l}\text { Considera que cumple con las funciones } \\
\text { establecidas en los instrumentos de } \\
\text { gestión. }\end{array}$ & 16 & $41.0 \%$ & 3 & $7.7 \%$ & 20 & $51.3 \%$ \\
\hline & $\begin{array}{l}\text { Considera que su productividad se ha } \\
\text { incrementado en este año. }\end{array}$ & 25 & $64.1 \%$ & 8 & $20.5 \%$ & 6 & $15.4 \%$ \\
\hline & $\begin{array}{l}\text { Recibe algún incentivo para mejorar la } \\
\text { productividad. }\end{array}$ & 36 & $92.3 \%$ & 1 & $2.6 \%$ & 2 & $5.1 \%$ \\
\hline \multirow{5}{*}{ Eficiencia } & $\begin{array}{l}\text { Cumple su meta descrita en el POI en el } \\
\text { tiempo establecido }\end{array}$ & 32 & $82.1 \%$ & 1 & $2.6 \%$ & 6 & $15.4 \%$ \\
\hline & $\begin{array}{l}\text { Considera que los recursos asignados se } \\
\text { dan en el tiempo establecido. }\end{array}$ & 35 & $89.7 \%$ & 0 & $0.0 \%$ & 4 & $10.3 \%$ \\
\hline & $\begin{array}{l}\text { Considera que los recursos asignados } \\
\text { son suficientes para cumplir con las } \\
\text { actividades establecidas en el POI. }\end{array}$ & 20 & $51.3 \%$ & 6 & $15.4 \%$ & 13 & $33.3 \%$ \\
\hline & $\begin{array}{l}\text { Cumple con los plazos establecidos en el } \\
\text { TUPA. }\end{array}$ & 30 & $76.9 \%$ & 2 & $5.1 \%$ & 7 & $17.9 \%$ \\
\hline & $\begin{array}{l}\text { Se encuentra comprometido con las } \\
\text { actividades que realiza. }\end{array}$ & 27 & $69.2 \%$ & 4 & $10.3 \%$ & 8 & $20.5 \%$ \\
\hline $\begin{array}{l}\text { Relación } \\
\text { interpersonal }\end{array}$ & $\begin{array}{l}\text { El ambiente de trabajo en la } \\
\text { municipalidad es agradable }\end{array}$ & 29 & $74.4 \%$ & 4 & $10.3 \%$ & 6 & $15.4 \%$ \\
\hline
\end{tabular}


Se comunica con sus compañeros de

$\begin{array}{llllllll}\text { trabajo para realizar las labores } & 17 & 43.6 \% & 10 & 25.6 \% & 12 & 30.8 \%\end{array}$ planificadas.

Considera que la comunicación entre

$\begin{array}{lllllllll}\text { compañeros ayuda a mejorar las } 11 & 28.2 \% & 8 & 20.5 \% & 20 & 51.3 \%\end{array}$ relaciones interpersonales.

Fuente: Cuestionario aplicado a los colaboradores de la municipalidad provincial Rioja.

\section{Interpretación:}

En la tabla 3 se caracteriza el desempeño laboral de los colaboradores desde la percepción del mismo colaborador, evidenciándose que, en la dimensión de eficacia, el 92.3\% (36) están "totalmente en desacuerdo" o "desacuerdo" que reciben algún incentivo para mejorar su productividad, el 84.6\% (29) le socializaron el MOF, ROF, RIT, MAPRO u otros, que cumplen con los objetivos planificados en el POI en un 74.4\% (29). Asimismo, que cuentan con los recursos necesarios para cumplir con los objetivos planificados en el POI, 69.2\% (27), que su productividad se ha incrementado en este año $64.1 \%$ (25), que se socializa al inicio del año los objetivos planificados en el POI, 53.8\% (21) y el $41.0 \%$ (16) cumple con las funciones establecidas en los instrumentos de gestión.

En la dimensión eficiencia, los colaboradores indican estar "totalmente en desacuerdo" o "desacuerdo" en un 89.7\% (35) que los recursos asignados se dan en el tiempo establecido, que cumplen su meta descrita en el POI en el tiempo establecido, 82.1\% (32), que cumplen con los plazos establecidos en el TUPA 76.9\% (30). Además, el 69.2\% (27) que se encuentran comprometidos con las actividades que realizan y el $51.3 \%$ (20) que los recursos asignados son suficientes para cumplir con las actividades establecidas en el POI.

En la dimensión relación interpersonal los colaboradores están "totalmente en desacuerdo" o "desacuerdo "en un 74.4\% (29) que el ambiente de trabajo en la municipalidad es agradable, que se comunican con sus compañeros de trabajo para realizar las labores planificadas $43.6 \%$ (17) y que la que la comunicación entre compañeros ayuda a mejorar las relaciones interpersonales $28.2 \%$ (11).

\section{Tabla 4}

Gestión por competencias desde la percepción del funcionario

\begin{tabular}{lllllll}
\hline Indicadores & \multicolumn{1}{c}{ TED /ED } & I & & \multicolumn{2}{c}{ DA / TDA } \\
& fi & $\%$ & fi & $\%$ & fi & $\%$
\end{tabular}




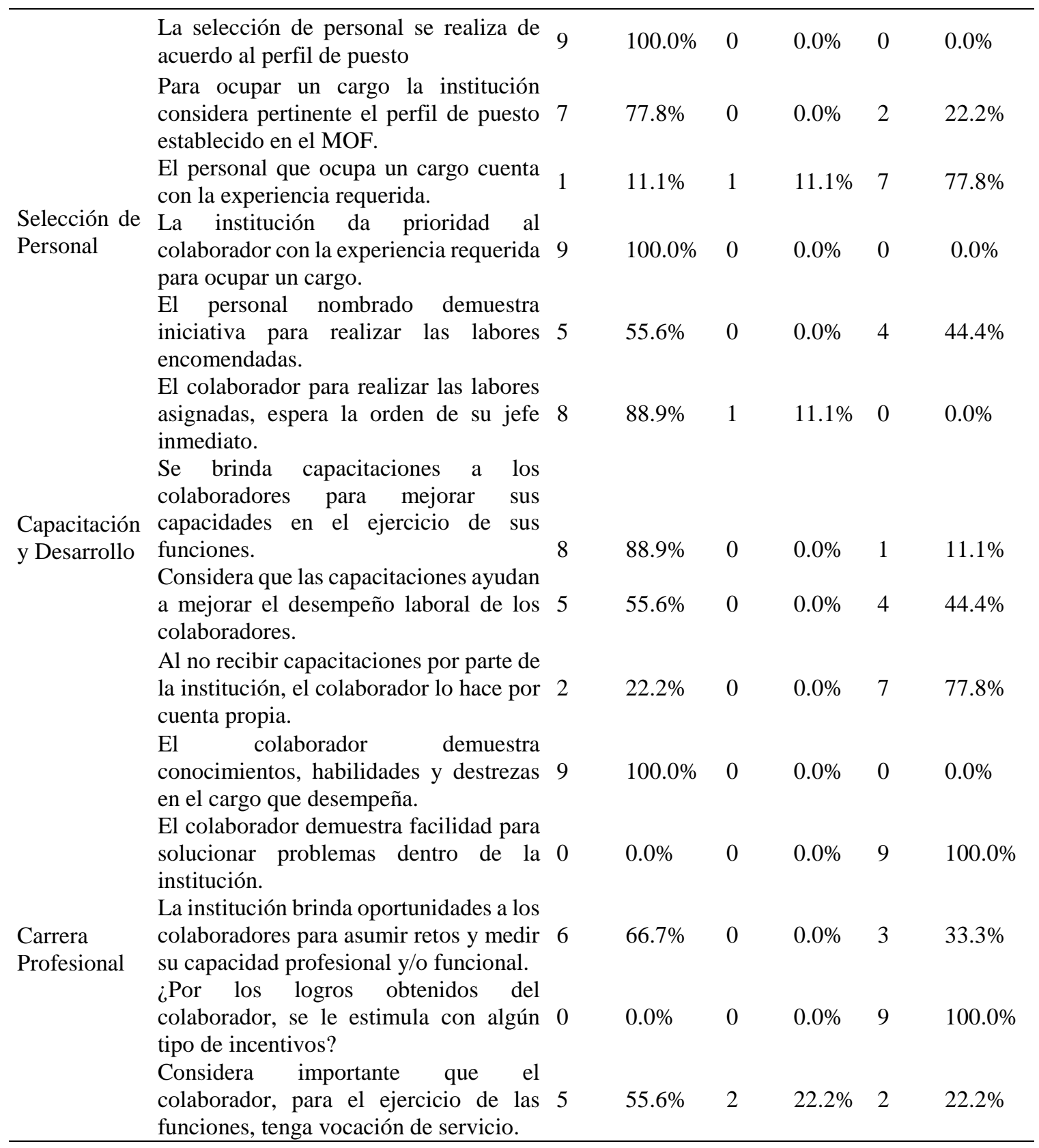

Fuente: Cuestionario aplicado a funcionarios de la municipalidad provincial Rioja.

\section{Interpretación:}

En la tabla 4 se caracteriza la gestión por competencias de los colaboradores desde la percepción del funcionario, en la dimensión selección de personal se evidencia que el 100\% (9) están "totalmente en desacuerdo" o "desacuerdo" que en la selección de personal se realiza de acuerdo al perfil de puesto, que la institución da prioridad al colaborador con la experiencia requerida para ocupar un cargo, el 89.9\% (8) indican que el colaborador para realizar las labores asignadas, espera la orden de su jefe inmediato, 
un 77.8\% (7) que para ocupar un cargo la institución no considera pertinente el perfil de puesto establecido en el MOF, además el 55.6\% (5) aprecian que el personal nombrado no demuestra iniciativa para realizar las labores encomendadas.

En la dimensión de capacitación y desarrollo, los funcionarios dicen estar "totalmente en desacuerdo" o "desacuerdo" en un 100. \% (9) que el colaborador demuestra conocimientos, habilidades y destrezas en el cargo que desempeña, que se brinda capacitaciones a los colaboradores para mejorar sus capacidades en el ejercicio de sus funciones $88.9 \%$ (8), que las capacitaciones ayudan a mejorar el desempeño laboral de los colaboradores $55.6 \%$ (5) y que, al no recibir capacitaciones por parte de la institución, el colaborador lo hace por cuenta propia $22.2 \%$ (2).

En la dimensión de carrera profesional, los funcionarios indican estar "totalmente en desacuerdo" o "desacuerdo" en un 66.7\% (6) que la institución brinda oportunidades a los colaboradores para asumir retos y medir su capacidad profesional y/o funcional y el $55.6 \%$ (5) que considera importante que el colaborador, para el ejercicio de las funciones, tenga vocación de servicio.

\section{Tabla 5}

Gestión por competencias desde la percepción del colaborador

\begin{tabular}{|c|c|c|c|c|c|c|c|}
\hline \multirow{2}{*}{ Indicadores } & & \multicolumn{2}{|c|}{ TED /ED } & \multicolumn{2}{|l|}{$\mathbf{I}$} & \multicolumn{2}{|c|}{ DA / TDA } \\
\hline & & $\mathbf{f i}$ & $\%$ & fi & $\%$ & fi & $\%$ \\
\hline \multirow{6}{*}{$\begin{array}{l}\text { Selección de } \\
\text { Personal }\end{array}$} & $\begin{array}{l}\text { Consideras que la selección de personal } \\
\text { se realiza de acuerdo al perfil de puesto. }\end{array}$ & 29 & $74.4 \%$ & 5 & $12.8 \%$ & 5 & $12.8 \%$ \\
\hline & $\begin{array}{l}\text { Para ocupar un cargo la institución } \\
\text { considera pertinente el perfil de puesto } \\
\text { establecido en el MOF. }\end{array}$ & 17 & $43.6 \%$ & 11 & $28.2 \%$ & 11 & $28.2 \%$ \\
\hline & $\begin{array}{l}\text { Considera que el personal que ocupa un } \\
\text { cargo cuenta con la experiencia } \\
\text { requerida. }\end{array}$ & 21 & $53.8 \%$ & 7 & $17.9 \%$ & 11 & $28.2 \%$ \\
\hline & $\begin{array}{l}\text { Considera que la institución da prioridad } \\
\text { al colaborador con la experiencia } \\
\text { requerida para ocupar un cargo. }\end{array}$ & 33 & $84.6 \%$ & 3 & $7.7 \%$ & 3 & $7.7 \%$ \\
\hline & $\begin{array}{l}\text { Demuestra iniciativa para realizar las } \\
\text { labores encomendadas. }\end{array}$ & 16 & $41.0 \%$ & 3 & $7.7 \%$ & 20 & $51.3 \%$ \\
\hline & $\begin{array}{l}\text { Esperas la orden de tu jefe inmediato } \\
\text { para realizar tus labores. }\end{array}$ & 25 & $64.1 \%$ & 8 & $20.5 \%$ & 6 & $15.4 \%$ \\
\hline \multirow[t]{3}{*}{$\begin{array}{l}\text { Capacitación } \\
\text { y Desarrollo }\end{array}$} & $\begin{array}{l}\text { Se te capacita para mejorar tus } \\
\text { capacidades en el ejercicio de tus } \\
\text { funciones. }\end{array}$ & 32 & $82.1 \%$ & 1 & $2.6 \%$ & 6 & $15.4 \%$ \\
\hline & $\begin{array}{l}\text { Consideras que las capacitaciones } \\
\text { ayudan a mejorar tu desempeño laboral. }\end{array}$ & 28 & $71.8 \%$ & 5 & $12.8 \%$ & 6 & $15.4 \%$ \\
\hline & $\begin{array}{l}\text { Al no recibir capacitaciones por la } \\
\text { municipalidad, lo realizas por cuenta } \\
\text { propia. }\end{array}$ & 12 & $30.8 \%$ & 6 & $15.4 \%$ & 21 & $53.8 \%$ \\
\hline
\end{tabular}




\begin{tabular}{|c|c|c|c|c|c|c|c|}
\hline \multirow{4}{*}{$\begin{array}{l}\text { Carrera } \\
\text { Profesional }\end{array}$} & $\begin{array}{l}\text { Demuestras conocimientos, habilidades } \\
\text { y destrezas en el cargo que desempeñas. } \\
\text { Demuestras facilidad para solucionar } \\
\text { problemas dentro de la institución. }\end{array}$ & 34 & $87.2 \%$ & 7 & $17.9 \%$ & 29 & $12.8 \%$ \\
\hline & $\begin{array}{l}\text { La institución te da la oportunidad de } \\
\text { asumir retos como para medir tú } \\
\text { capacidad profesional y/o funcional. }\end{array}$ & 29 & $74.4 \%$ & 4 & $10.3 \%$ & 6 & $15.4 \%$ \\
\hline & $\begin{array}{l}\text { Te estimulan por los logros obtenidos } \\
\text { con algún tipo de incentivos. }\end{array}$ & 17 & $43.6 \%$ & 10 & $25.6 \%$ & 12 & $30.8 \%$ \\
\hline & $\begin{array}{l}\text { Para el ejercicio de tus funciones } \\
\text { consideras que es necesario tener } \\
\text { vocación de servicio. }\end{array}$ & 11 & $28.2 \%$ & 8 & $20.5 \%$ & 20 & $51.3 \%$ \\
\hline
\end{tabular}

Fuente: Cuestionario aplicado a los colaboradores de la municipalidad provincial Rioja.

\section{Interpretación:}

En la tabla 5 se caracteriza la gestión por competencias de los colaboradores desde la percepción del mismo colaborador, evidenciándose que en la dimensión selección de personal, están "totalmente en desacuerdo" o "desacuerdo" en un 84.6\% (33) que la institución da prioridad al colaborador con la experiencia requerida para ocupar un cargo, que en la selección de personal se realiza de acuerdo al perfil de puesto 74.4\% (29), que esperan la orden de tu jefe inmediato para realizar tus labores $64.1 \%$ (25), que el personal que ocupa un cargo cuenta con la experiencia requerida $53.8 \%$ (21), además que para ocupar un cargo la institución considera pertinente el perfil de puesto establecido en el MOF 43.6\% (17).

En la dimensión de capacitación y desarrollo, los colaboradores indican estar "totalmente en desacuerdo" o "desacuerdo" en un 87.2\% (34) que demuestran conocimientos, habilidades y destrezas en el cargo que desempeñan, que se les capacita para mejorar tus capacidades en el ejercicio de tus funciones $82.1 \%$ (32), que las capacitaciones ayudan a mejorar tu desempeño laboral en un $71.8 \%$ (28), al no recibir capacitaciones por la municipalidad, lo realizan por cuenta propia $30.8 \%$ (12).

En la dimensión de carrera profesional, los colaboradores están "totalmente en desacuerdo" o "desacuerdo" en 74.4\% (29) que la institución les da la oportunidad de asumir retos para medir sus capacidades profesionales y/o funcionales, que se les estimulan por los logros obtenidos con algún tipo de incentivos $43.6 \%$ (17) y que para el ejercicio de sus funciones es necesario tener vocación de servicio 28.2\% (11).

\section{DISCUSIÓN}


La presente investigación tuvo como objetivo, identificar el nivel del desempeño laboral de los colaboradores de la Municipalidad Provincial de Rioja, 2020. Los resultados describen, que, según la percepción de los funcionarios y colaboradores, el nivel de desempeño laboral de estos últimos es "Bajo" (77.8\% y 66,7\%) respectivamente. Lo que indica que en dicha entidad los trabajadores no están cumpliendo con las metas establecidas, falta de compromiso con las asignaciones de responsabilidades y escasa comunicación asertiva entre ellos.

En tal sentido, Grijalva, Castro y Guamán (2019), tienen coincidencia con los resultados de la investigación donde el desempeño laboral es "muy malo" (82\%) según la percepción de los clientes; infiriendo que la actitud, personalidad, percepción y aprendizaje están asociadas. Pudo constatar que el desempeño de los trabajadores influye en la calidad de trabajo, siendo muy importante la predisposición que muestren para cumplir sus tareas. Asimismo, el individualismo y la baja remuneración afecta el clima organizacional y el rendimiento laboral. Considera que, el trabajo en equipo es un factor significativo para el desarrollo de las capacidades laborales, habilidades e innovación.

Del mismo modo, Del Toro, Salazar y Gómez (2011), muestran que el 58\% de los usuarios perciben que el desempeño laboral de los trabajadores es "deficiente", lo que imposibilita el logro de los objetivos trazados y el uso adecuado de los recursos. Es importante resaltar que, el deficiente desempeño laboral se manifiesta según las actitudes y aptitudes de los trabajadores, influyendo negativamente en su trabajo y a su vez de la organización.

A diferencia de, Díaz, Isla, Hernández y Rosales (2012), el 75\% mencionaron que el desempeño laboral es óptimo, es decir, que los trabajadores cumplen con sus labores de manera responsable, se adaptan con facilidad a los cambios, asumen retos y están acostumbrados a trabajar bajo presión para alcanzar las metas en el tiempo establecido. Debido tal vez a que son estimulados constantemente.

Con respecto a la caracterización del desempeño laboral de los colaboradores, desde la percepción del funcionario y los trabajadores, en la dimensión eficacia, la mayoría mencionó estar en desacuerdo, ya que no se cumplen, ni cuentan con los recursos necesarios para realizar con los objetivos planificados en el POI, ni socializan el MOF, ROF, RIT, MAPRO. Asimismo, en la dimensión eficiencia, la mayoría manifestó estar totalmente en desacuerdo, que no cumplen sus metas en función y con los plazos 
establecidos en el TUPA y en la falta de compromiso en las actividades que realizan. En la dimensión interpersonal, expresaron su desacuerdo, debido al ambiente de trabajo poco agradable y la falta de comunicación empática ente los trabajadores.

Por su parte, Salgado (2011), presenta resultados similares, ya que, la mayoría expresó estar totalmente en desacuerdo con el trabajo que realizan los empleados, por la falta de eficacia y efectividad en su rendimiento. Para este autor un buen desempeño es sinónimo de eficacia y eficiencia, así como otras acciones que son relevantes para alcanzar las metas de la organización y puede medirse en función al nivel de contribución de cada individuo, la efectividad se refiere a la evaluación de los resultados del desempeño y está fuera de la influencia del colaborador.

En opinión de, Sanabria (2015), los resultados obtenidos indican que la mayoría no está de acuerdo con el desempeño laboral, porque no alcanzan las metas establecidas en su plan anual de trabajo. Puntualizó, que, uno de los requisitos más importantes del capital humano es su eficacia y eficiencia, para lograr conseguir su máxima productividad; asimismo enfocarse a políticas que direccionen al desempeño laboran alineados a los objetivos organizacionales y finalmente se debe mantener equilibrio en los intereses del personal, que incluya las recompensas, motivación y un nivel de seguridad en la continuidad laboral en la organización.

Sin embargo, Reinoso y Pérez (2019), partiendo de sus resultados, donde la mayoría demostraron su disconformidad con el rendimiento laboral, llegaron a la conclusión, que, tiene que ver mucho la actitud para ofrecer un producto y un servicio de calidad, se requiere que los trabajadores brinden una buena atención al cliente, que exista la predisposición para realizar sus actividades con eficiencia cumpliendo asertivamente con los procedimientos para el empleado y la Administración. Desde el punto de vista competitivo los trabajadores de la empresa pueden adaptarse a los cambios con total normalidad. Puesto que la innovación permite dotarse de recursos técnicos mejorar y modernizar la presentación del producto, infraestructura y el servicio.

Con respecto a la caracterización de la gestión por competencias en la dimensión selección del personal, la mayoría mencionó estar totalmente en desacuerdo, que para la selección de personal no toman en cuenta el perfil del puesto, ni la experiencia requerida. En cuanto a la dimensión capacitación y desarrollo, la mayoría opinó estar en desacuerdo, que las capacitaciones ayuden a mejorar el desempeño laboral de los colaboradores, ya 
que no demuestran poseer mayor conocimiento ni destrezas en el cargo que desempeñan. Asimismo, en la dimensión carrera profesional, expresaron estar totalmente en desacuerdo, porque la institución no da oportunidad de asumir retos como para su capacidad profesional y/o funcional, ni existen estímulos por los logros laborales obtenidos.

Es por ello, Díaz (2018), manifiesta estar en desacuerdo con los procesos de contratación que realiza la institución; evidenciando que no se toma en cuenta las competencias del trabajador, la mayoría de los puestos laborales son ocupados por favores políticos, saltando las normas establecidas en la gestión por competencias. En cambio, si se aplicaría o se tomara en cuenta las capacidades y competencias de cada trabajador, la selección de personal sería transparente. Los trabajadores asistirían a capacitaciones de acuerdo al rubro de desenvolvimiento y los resultados serían más óptimos.

En cambio, Santacruz (2019), expresó sobre los modelos de gestión por competencias hacen una incorporación y articulación entre los procesos de selección, evaluación del desarrollo y capacitación; con el objetivo de que las organizaciones logren administrar, potencializar y desarrollar acertadamente su talento humano, debido que son tomadas como "una creación desde una conjunción de elementos (conocimiento, entender llevar a cabo, características o aptitudes), y elementos del ámbito (relaciones, documentos, aclaraciones y otros) que son movilizados para conseguir un buen desarrollo.

Finalmente, se podría indicar que, el desempeño laboral es deficiente en nuestro estudio y en la de otros investigadores, y que su mejora requiere de capacitación, motivación y estímulos; siendo el desarrollo de competencias la más importante. Es por ello que, para responder a esa necesidad sentida, se presenta el modelo de gestión por competencias para optimizar el desempeño laboral de los colaboradores de una entidad municipal y elevar la productividad.

\section{CONCLUSIONES O CONSIDERACIONES FINALES}

En conclusión, podemos manifestar que:

1. El desempeño laboral de los colaboradores de la Municipalidad Provincial de Rioja es deficiente, por lo que es importante diseñar un modelo de gestión para desarrollar o fortalecer competencias cognitivas, habilidades y actitudinales optimizando su rendimiento, productividad y la administración de los recursos financieros y humanos. 
2. El nivel del desempeño laboral de los colaboradores de la Municipalidad provincial de Rioja, es "Bajo" según la percepción de los funcionarios y colaboradores; lo que indica, que en dicha institución los trabajadores no asumen con responsabilidad sus funciones, ocasionando que no se cumplan las metas establecidas.

3. El desempeño laboral de los colaboradores de la Municipalidad provincial de Rioja, es deficiente, puesto que no se cumplen con los objetivos planificados, los recursos asignados no se dan en el tiempo establecido, así como el ambiente laboral no es óptimo. 4. La gestión por competencias de los funcionarios de la Municipalidad provincial de Rioja, es deficiente, porque los puestos de trabajo son ocupados sin tener en cuenta el perfil que establece el MOF, no existe un plan de capacitación para los colaboradores y no se brinda oportunidad de crecimiento profesional.

\section{LISTA DE REFERENCIAS}

Bailón, N. (2014). La capacitación y el desarrollo del personal. [Blog] GestioPolis. Available at: https://www.gestiopolis.com/la-capacitacion-y-eldesarrollo-delpersonal/ [Accessed 2 Mar. 2018].

Bocanegra, J. (2018). Competencias expertas requeridas por las compañías en Tarapoto.

(Artículo científico). Universidad Peruana Unión, Tarapoto, Perú

Carhuachín, F. (2018). Administración por competencias para el desarrollo laboral del talento humano en la Municipalidad provincial de Otuzco - La Independencia, 2018. (Artículo científico). Universidad Señor de Sipán. Pimentel, Perú

Champagnet, U. (05 de 03 de 2001). Licenciatura en RR.HH. Recuperado de: Gestiopolis: http://www.gestiopolis.com/desarrollo-profesional

Chávez, N. (2012). La gestión por competencias y ejercicio del coaching empresarial, dos estrategias internas para la organización. Pensamiento \& Gestión (33), 140-161. Recuperado de http://www.redalyc.org/articulo.oa?id=64624867007

Chiavenato, I. (2013). Comportamiento Organizacional. Editorial Mc. Graw Hill, Méxic

Correa, St. (2017). Incidencia de la Gestión por competencias en el crecimiento empresarial. (Artículo científico). Universidad Técnica de Machala, Ecuador. DOI: https://doi.org/10.33890/innova.v2.n1.2017.76

Da Silva, R. (2008). Recuperado de http://www.promonegocios.net/administracion/definicioneficacia.html

Dessler, G. (2001). Administración de Personal. México: Pearson Educación 
Díaz, J. (2018). Influencia de las competencias gerenciales y la gestión por resultados en la imagen institucional. (Artículo científico). Universidad César Vallejo, Lima, Perú.

Dorta, J. y Verona, M. (2006). Concepto, perspectivas y medida del crecimiento empresarial. Cuaderno de Administración, vol. 19, núm. 31, pp. 165-195. Pontificia Universidad Javeriana, Bogotá, Colombia. Recuperado en: http://www.redalyc.org/artículo.oa?id=20503107

García, M., García, A., Pérez, R., González, M., \& Martínez, Y. (2014). La gestión por competencias para el desempeño exitoso, análisis desde el sector empresarial cubano. Revista cientifican avances, 16(1), 64-75. Recuperado de https://dialnet.unirioja.es/servlet/articulo?codigo=5350913

García, Y., Reyes, L., \& Javier, C. (2009). ¿Por qué la importancia de implementar Sistemas de Gestión por Competencias en nuestras organizaciones? Ciencias Holguín, $\quad$ XV(2), 1-9. Recuperado de http://www.redalyc.org/articulo.oa?id=181517990005

García, Y., Reyes, L., \& Javier, C. (2009). ¿Por qué la importancia de implementar Sistemas de Gestión por Competencias en nuestras organizaciones? Ciencias Holguín, $\quad X V(2), \quad 1-9 . \quad$ Recuperado de http://www.redalyc.org/articulo.oa?id=181517990005

González, M. (2000). De la gestión por competencias a la gestión por competencias personales, hacia un nuevo modelo de gestión de personas en la era del conocimiento. Ciencia y Sociedad, XXV(1), 35-61. Recuperado de: http://www.redalyc.org/articulo.oa?id=87011348002

Guerrero, C., Valverde, M., \& Tatiana, M. (2013). Un análisis de la gestión por competencias en la empresa española. De la teoría a la práctica. Contaduría y Administración, 58(1), 251-288. Recuperado de: http://www.redalyc.org/articulo.oa?id=39525580010

Hernández, V. (2018). Modelo de administración de talento humano por competencias de la ONG para la discapacidad fundación cuesta Holguín. (Artículo científico). Pontificia Universidad Católica de Ambato, Ecuador.

Lozano, G. y Mendoza, M. (2018), Relación de la administración por competencias con el desarrollo laboral de los ayudantes en una municipalidad de la zona San 
Martín - Perú. (Tesis de posgrado). Universidad Peruana la Unión. Tarapoto, Perú

Mandamiento, A. (2019). Administración por competencias y desarrollo laboral del personal de la Municipalidad provincial de Candarave. (Artículo científico). Universidad Privada de Tacna, Perú 